\title{
Continuous interscalene brachial plexus block
}

I NTERSCALENE blockade (ISB) of the brachial plexus is well suited for shoulder surgery as the sole anesthetic technique or in combination with general anesthesia. A success rate of $87-100 \%$ is reported when single shot ISB is performed by either paresthesia or nerve stimulator technique. However, if regional analgesia beyond $12-24 \mathrm{hr}$ is warranted, it is necessary to perform continuous ISB whereby local anesthetic is infused continuously through an accurately placed catheter in the interscalene groove. Because catheter insertion and fixation is technically challenging, continuous ISB technique has a failure rate of up to $25 \% .{ }^{1}$ In this issue of the Journal, Boezaart describes a "new" technique of catheter placement and fixation with the goal of improving the success rate of continuous ISB. ${ }^{2}$ Several features of this new technique deserve attention and comments.

Boezaart's approach to the interscalene groove relies on the placement of a Tuohy needle halfway between the mastoid and clavicle and posterior to the clavicular head of the sternocleidomastoid muscle. The needle is then advanced directly caudad and parallel to the vertebral column. This approach closely resembles Winnie's subclavian perivascular approach to the brachial plexus although the needle entry site for ISB is higher up. In this case, the Tuohy needle and eventually the indwelling catheter traverse nerve roots of the brachial plexus in a cephalad to caudad direction. This may potentially increase the contact surface with the brachial plexus as opposed to the classical perpendicular approach used for single shot ISB. Increasing the length of catheter dwelling in the interscalene groove may also decrease the risk of catheter dislodgment.

It is also important to note that directing the needle cephalad-caudad may avoid potential life threatening complications. As the needle points away from the cervical transverse process and intervertebral foramina, unintentional needle and catheter entry and local anesthetic injection into the vertebral artery ${ }^{3}$ and epidural space ${ }^{4}$ can be avoided.

Boezaart uses a special indwelling catheter (Theracath, Arrow) for continuous ISB with an inner stylet and a metal tip that permits electrical conduction. This invaluable feature allows nerve stimulation through the catheter to confirm the accuracy of the final indwelling catheter position. Conventional catheter insertion techniques using a nerve stimulator (e.g. Contiplex, B. Braun Medical) only confirm correct placement of the needle in the interscalene groove and not the catheter. For this reason, it is possible for the threaded catheter to pass the desired target of blockade entirely unnoticed. Regrettably, if this occurrence is not detected until after needle removal and local anesthetic injection, a repeat block procedure is necessary.

Boezaart advocates subcutaneous tunneling of the indwelling catheter as a means of decreasing the chance of catheter dislodgment as opposed to conventional fixation methods using adhesive tape and suture. The concept of subcutaneous tunneling is not new; a similar technique is commonly applied in chronic epidural catheterization to prevent inadvertent displacement and infection. To do so, Boezaart advocates inserting the tunneling needle at the suprasternal notch and advancing the needle subcutaneously and superolaterally toward the catheter entry site before exiting the skin next to the catheter. In our opinion, this manner of tunneling presents inherent danger since the sharp end of the Tuohy needle may cut into and damage the catheter. We recommend a potentially safer technique of catheter tunneling. First, the Tuohy needle is partially withdrawn over the body of the catheter once the interscalene catheter is correctly positioned. This ensures protection of the catheter within the needle shaft. Second, a \#2 intravenous angiocatheter of sufficient size (e.g., 16 gauge) is used to create a separate subcutaneous tunnel and advance the tip until it exits skin next to the Tuohy needle entry site. Then the Tuohy needle is removed and the needle portion of the angiocatheter. Finally, the exterior end of the interscalene catheter is threaded through the tip of the plastic cannula before pulling out the cannula completely. The interscalene catheter is now tunneled subcutaneously. This technique of tunneling will eliminate potential catheter shearing or cutting. 
Ipsilateral phrenic nerve block is common after ISB possibly as a result of local anesthetic spreading to upper cervical nerve roots $\left(\mathrm{C}_{3}, \mathrm{C}_{4}\right)$ or anteriorly to block phrenic nerve in front of the anterior scalene muscle. Although clinical manifestation of phrenic nerve block is uncommon, it is important to remember that significant respiratory dysfunction and hypoxemia may occur in geriatric patients ${ }^{5}$ and those with preexisting borderline respiratory reserve.

Urmey has shown ultrasonographic evidence of hemidiaphragmatic paralysis resulting in paradoxical movement in $100 \%$ of the patients receiving $25-40$ $\mathrm{mL}$ mepivacaine. ${ }^{6}$ In Boezaart's study, it is surprising and puzzling to see a considerable difference in the incidence of phrenic nerve block when different nerve localization and local anesthetic injection methods were used. After administration of $20 \mathrm{~mL}$ bupivacaine $0.5 \%$, the incidence was $85 \%$ with the paresthesia technique, $35 \%$ with the nerve stimulator technique and only $20 \%$ when the local anesthetic was injected through the catheter. These findings disagree with those of previous studies which show consistent ipsilateral diaphragmatic paresis when $20-28 \mathrm{~mL}$ bupivacaine $0.75 \%$ were administered. ${ }^{7}$ In a volunteer study, we have also demonstrated hemidiaphragmatic paralysis in $80 \%$ of the subjects receiving only $10 \mathrm{~mL}$ of bupivacaine $0.5 \%$ and in $17 \%$ of those who received 10 $\mathrm{mL}$ of bupivacaine $0.25 \%{ }^{8}$ Perhaps, Boezaart's method of insertion in a caudad direction places the catheter more distally in the brachial plexus sheath and further away from the $\mathrm{C}_{3-5}$ nerve roots.

The success of ISB to provide anesthesia during shoulder surgery is dose dependent. Most commonly $40 \mathrm{~mL}$ of a local anesthetic e.g. 1.5\% mepivacaine are required not only to provide surgical anesthesia to the shoulder but also muscle paralysis to the rest of the upper extremity. Administration of a lower volume, 20 $\mathrm{mL}$ bupivacaine $0.5 \%$, as used in Boezaart's study will likely provide preferential analgesia to the shoulder without complete anesthesia to the $\mathrm{C}_{8}-\mathrm{T}_{1}$ nerve roots. One may speculate that Boezaart's continuous ISB catheter technique alone is not reliably sufficient for surgical anesthesia without supplementation of general anesthesia. However, if selective analgesia is the goal, the value of ISB using $10 \mathrm{~mL}$ bupivacaine $0.125 \%$ and bupivacaine $0.25 \%$ following arthroscopic and open shoulder procedures respectively has been demonstrated. .,10 $^{9}$

Although not a new technique, perioperative data comparing continuous ISB with other analgesic methods are limited. Most commonly, an infusion of bupivacaine $0.25 \%$ at a starting rate of $0.25 \mathrm{mg} \cdot \mathrm{kg}^{-1} \cdot \mathrm{hr}^{-1}$ is recommended. Two prospective studies recently con- ducted by Borgeat showed that patients receiving continuous ISB through a patient controlled analgesia method (PCIA) experienced superior analgesia and diminished side effects following major shoulder surgery compared with those who received patient controlled iv opioid. ${ }^{11,12}$ Patients in both treatment groups received ISB prior to endotracheal general anesthesia but those in the PCIA group (20 patients) were maintained with bupivacaine $0.15 \%$ at $5 \mathrm{ml} \cdot \mathrm{hr}^{-1}$ plus PCA bolus $3.4 \mathrm{~mL}$ every 20 min as required. ${ }^{11}$ Pain scores were lower 12 and $18 \mathrm{hr}$ during PCIA treatment and vomiting and pruritus did not occur. A similar degree of analgesic efficacy was demonstrated in another study when ropivacaine $0.2 \%$ was used for PCIA in 30 patients. ${ }^{12}$ Motor block was minimal, patient satisfaction was higher and incomplete analgesia was adequately supplemented with paracetamol during PCIA. Technical failure was found in $10 \%$ of the PCIA patients.

When is continuous ISB indicated? Additional cost, time and technical challenge associated with the technique may argue that continuous ISB be reserved for selected patients. For example, patients requiring intensive physiotherapy after major shoulder surgery (especially redo surgery) or those with chronic upper extremity pain as with complex regional pain syndrome may benefit from continuous ISB analgesia. Continuous ISB has also been used successfully to treat cancer patients suffering from bone pain due to humeral metastasis. ${ }^{13}$ Furthermore, continuous ISB can be used as part of a novel analgesic strategy allowing painful inpatient shoulder procedures (e.g., rotator cuff repair) to be done as outpatient procedures. Such a novel approach has recently been described by Rawal whose preliminary study demonstrated that patient controlled local anesthetic administration at home after surgery can be both effective and safe for a variety of orthopedic procedures. ${ }^{14}$ However, before widespread enthusiastic endorsement of local anesthetic administration at home, perioperative outcome data confirming patient safety must be gathered in future large scale prospective studies.

Although generally accepted in children, performing ISB in adult patients under general anesthesia, as described by Boezaart, remains a controversial subject and is often dependent upon institutional or national practice standard and attitudes. Recent data indicate that paresthesia during needle placement and pain during local anesthetic injection are two key elements closely related to the occurrence of serious neurological injury.$^{15}$ It is logical to assume that these warning signs will be missed in an unconscious patient. Furthermore, preliminary data show that nerve stimulator guided 
nerve block may not be as safe as once thought. Urmey showed that sensory paresthesia may be elicited without consistently getting muscle twitching in $25 \%$ of the patients when stimulated at $1 \mathrm{~mA}$ for ISB suggesting a discrepancy between motor and sensory response elicited by needle puncture. ${ }^{16}$ Thus, a lack of motor response does not rule out needle proximity to nerve tissue. Several cases of neuropathy have been reported after the use of a nerve stimulator for ISB on patients under general anesthesia, one leading to permanent neurological deficit. ${ }^{17}$ In my opinion, the decision to perform regional anesthesia in a heavily sedated or anesthetized patient should not be made indiscriminately. This is especially true for procedures such as total shoulder arthroplasty in which brachial plexus injury as a surgical complication is a possibility. This may leave the anesthesiologist open for incrimination.

In summary, continuous ISB is a useful technique to provide regional analgesia for a variety of shoulder conditions as described. The new technique described by Boezaart will likely improve the accuracy of ISB catheter placement and decrease the chance of dislodgment. However, the practice of performing ISB routinely in patients under general anesthesia cannot be endorsed.

\section{Bloc interscalène con- tinu du plexus brachial}

Le bloc interscalène (BIS) du plexus brachial convient bien à la chirurgie de l'épaule, employé seul ou avec l'anesthésie générale. Son taux de réussite est d'ailleurs de $87-100 \%$ en injection unique selon la technique de la paresthésie ou du neurostimulateur. Cependant, si l'analgésie locorégionale est justifiée pour une période au delà de 12-24 h, il est nécessaire de procéder à un BIS continu par lequel l'anesthésique local est perfusé au moyen d'un cathéter correctement placé dans le sillon interscalène. Étant donné le défi technique de l'insertion et de la fixation du cathéter, le BIS continu connaît un taux d'échec qui peut aller jusqu'à $25 \%{ }^{1}$ Boezaart décrit, dans le présent numéro du Journal, une «nouvelle» technique qui pourrait augmenter les chances de succès de la mise en place et de la fixation du cathéter. ${ }^{2}$ Quelques particularités méritent notre attention et nos commentaires.

Pour atteindre le sillon interscalène, Boezaart propose l'insertion d'une aiguille Tuohy à mi-chemin entre la mastoïde et la clavicule et derrière le chef claviculaire du muscle sterno-cléido-mastoïdien, puis en direction caudale et parallèle à la colonne vertébrale. Cette méthode ressemble beaucoup à l'approche du plexus brachial de Winnie, périvasculaire et sous-clavière, mais le point de ponction du BIS est plus haut. Dans ce cas, l'aiguille Tuohy, et finalement le cathéter à demeure, traverse les racines nerveuses du plexus brachial en direction caudale. Cette façon de procéder peut accroître la surface de contact avec le plexus brachial contrairement à l'approche perpendiculaire traditionnelle utilisée pour la dose unique d'un BIS. En outre, la pose d'un cathéter avec une telle surface de contact dans le sillon intersaclène peut diminuer le risque de déplacement.

Il est aussi important de noter que l'insertion de l'aiguille en direction caudale peut prévenir d'éventuelles complications dangereuses. L'aiguille étant insérée loin de l'apophyse transverse cervicale et des trous de conjugaison, on peut éviter que sa mise en place et celle du cathéter ainsi que l'injection d'anesthésique local aient lieu dans l'artère vertébrale 3 et l'espace épidural ${ }^{4}$.

Boezaart utilise un cathéter à demeure spécial (Theracath, Arrow), comportant un stylet intérieur et une pointe de métal pour permettre la conduction électrique. C'est une caractéristique particulièrement intéressante qui permet la stimulation nerveuse au travers du cathéter pour confirmer la justesse de la position finale de ce dernier. Les techniques traditionnelles d'insertion des cathéters à l'aide d'un neurostimulateur (par ex. Contiplex, B. Braun Medical) ne font que confirmer la position exacte de l'aiguille dans le sillon interscalène, non celle du cathéter. C'est pourquoi l'insertion d'un cathéter au delà du point ciblé pour le bloc pourrait passer tout à fait inaperçue. Malheureusement, si cette erreur n'est pas détectée avant le retrait de l'aiguille et l'injection d'anesthésique local, il faut reprendre le bloc.

Pour diminuer le risque de déplacement du cathéter à demeure, Boezaart conseille la formation d'un canal sous-cutané, contrairement aux méthodes de fixation habituelles avec suture et ruban adhésif. Cette idée n'est certes pas nouvelle, puisqu'une technique semblable est couramment utilisée lors d'un cathétérisme épidural de longue durée pour prévenir un déplacement involontaire et une infection. Pour ce faire, Boezaart suggère d'insérer l'aiguille de tunnellisation à la fourchette sternale et de la pousser sous la peau et en direction supérolatérale vers le site d'entrée du cathéter avant de ressortir près du cathéter. Selon nous, cette méthode présente un danger inhérent, car la pointe tranchante de l'aiguille Tuohy pourrait pénétrer dans le cathéter et l'endommager. Nous recommandons une tunnellisation proba- 
blement plus sûre. D'abord, l'aiguille Tuohy est partiellement retirée au-dessus du corps du cathéter interscalène une fois qu'il est en position correcte, assurant ainsi la protection du cathéter à l'intérieur de l'aiguille. Ensuite, on utilise un angiocathéter intraveineux de taille suffisante (par ex. calibre 16) pour créer un canal sous-cutané séparé et on en pousse la pointe jusqu'à ce qu'elle ressorte près du point de ponction de l'aiguille Tuohy. L'aiguille Tuohy est alors retirée ainsi que la partie aiguille de l'angiocathéter. Enfin, l'extrémité extérieure du cathéter interscalène est introduite par la pointe de la canule de plastique avant de retirer complètement la canule. Le cathéter interscalène est maintenant dans un canal sous-cutané. Cette tunnellisation éliminera un cisaillement ou une coupure potentiels.

Le blocage du nerf phrénique ipsilatéral est fréquent après un BIS, résultat probable de la diffusion de l'anesthésique local vers les racines nerveuses cervicales supérieures $\left(\mathrm{C}_{3}, \mathrm{C}_{4}\right)$ ou vers l'avant pour bloquer le nerf phrénique en avant du muscle scalène antérieur. Malgré les évidences rares de blocage du nerf phrénique, il faut rappeler qu'une hypoxémie et une insuffisance respiratoire marquées peuvent survenir chez les patients âgés et chez ceux qui présentent une réserve respiratoire limite préexistante.

Urmey a montré à l'échographic l'évidence d'une paralysie hémidiaphragmatique provoquant un mouvement paradoxal chez $100 \%$ des patients qui avaient reçu $25-40 \mathrm{ml}$ de mépivacaïne. ${ }^{6}$ Dans l'étude de Boezaart, il est à la fois surprenant et embarrassant de constater une différence considérable de l'incidence de blocage du nerf phrénique selon différentes méthodes d'injection de l'anesthésique local et divers points de ponction du nerf. Après l'administration de $20 \mathrm{ml}$ de bupivacaïne $0,5 \%$, l'incidence était de $85 \%$ selon la technique de la paresthésie, $35 \%$ selon celle du neurostimulateur et seulement $20 \%$ lorsque l'anesthésique local était injecté au travers du cathéter. Ces résultats contredisent ceux d'études précédentes qui démontrent une parésie diaphragmatique homolatérale constante quand on administre de 20 à $28 \mathrm{ml}$ de bupivacaïne $0,75 \%{ }^{7}$ Une étude auprès de volontaires a montré aussi qu'une paralysie hémidiaphragmatique se produisait chez $80 \%$ des sujets qui avaient reçu seulement $10 \mathrm{ml}$ de bupivacaüne $0,5 \%$ et chez $17 \%$ de ceux qui en avaient reçu 10 $\mathrm{ml}$ d'une concentration de $0,25 \%{ }^{8}$ Peut-être que la méthode d'insertion de Boezaart en direction caudale fournit une position plus distale du cathéter dans la gaine du plexus brachial et plus éloignée des racines nerveuses de $\mathrm{C}_{3-5}$.

La réussite de l'anesthésie avec le BIS pendant la chirurgie de l'épaule dépend de la dose d'anesthésique reçue. Habituellement, il s'agit de $40 \mathrm{ml}$ d'un anesthésique local, par exemple $1,5 \%$ de mépivacaïne sont nécessaires pour fournir non seulement l'anesthésie pendant la chirurgie de l'épaule, mais aussi une relaxation musculaire du reste du membre supérieur. L'administration d'un volume plus faible, $20 \mathrm{ml}$ de bupivacaüne 0,5\%, comme dans l'étude de Boezaart, fournit probablement une analgésie préférentielle de l'épaule sans anesthésie complète des racines nerveuses de $\mathrm{C}_{8}-\mathrm{T}_{1}$. On peut douter du fait que la technique de Boezaart du cathéter à demeure pour le BIS continu employée seule soit suffisante pour assurer l'anesthésie chirurgicale, sans le complément de l'anesthésie générale. Toutefois, pour les cas où l'objectif est une analgésie sélective, on a démontré la valeur du BIS à base de $10 \mathrm{ml}$ de bupivacaïne $0,125 \%$ et de bupivacaïne $0,25 \%$ suivant l'arthroscopie et la chirurgie de l'épaule respectivement. ${ }^{9,10}$

Même si le BIS continu n'est pas une nouvelle technique, les études comparatives de ses données périopératoires avec celles d'autres méthodes analgésiques demeurent limitées. Le plus souvent, une perfusion de bupivacaïne 0,25\% dont le débit de départ est de 0,25 $\mathrm{mg} \cdot \mathrm{kg}^{-1} \cdot \mathrm{hr}^{-1}$ est recommandée. Borgeat a montré récemment par deux études prospectives que des patients qui reçoivent un BIS continu en utilisant une méthode d'analgésie contrôlée par le patient (AICP), bénéficient d'une analgésie supérieure et d'une réduction des effets secondaires par rapport à ceux qui reçoivent des opiacés iv en $A C P,{ }_{1}^{11,12}$ à la suite d'une chirurgie majeure de l'épaule. Les patients des deux groupes avaient reçu un BIS avant l'anesthésie générale endotrachéale, mais l'anesthésie des patients du groupe AICP (20 patients) a été maintenue avec de la bupivacaïne $0,15 \%$ à $5 \mathrm{ml} \cdot \mathrm{hr}^{-1}$ et un bolus ACP de $3-4 \mathrm{ml}$ à toutes les $20 \mathrm{~min}$ au besoin. ${ }^{11}$ Les scores de douleur étaient plus bas à $12 \mathrm{~h}$ et $18 \mathrm{~h}$ du traitement AICP et il n'y avait pas de vomissements ni de prurit. Un degré semblable d'efficacité analgésique a été démontré lors d'une autre étude à base de ropivacaïne $0,2 \%$ pour l'AICP chez 30 patients. ${ }^{12}$ Le blocage moteur a été minimal, la satisfaction du patient était plus élevée et on a complété l'analgésie inadéquate avec du paracétamol pendant l'AICP. Une défaillance technique a été détectée chez $10 \%$ des patients recevant une AICP.

Quelles sont les indications pour un BIS continu? On serait enclin à le réserver à des patients sélectionnés étant donné les frais supplémentaires, le temps et le défi technique associés à cette méthode. Par exemple, des patients qui ont besoin de physiothérapie intensive après une chirurgie majeure de l'épaule (surtout la chirurgie reprise) ou ceux qui souffrent de douleur chronique aux membres supérieurs, comme dans le cas 
de l'algodystrophie sympathique, peuvent bénéficier de son analgésie continue. Le BIS continu a aussi été utilisé avec succès pour soulager les douleurs osseuses reliées à des métastases humérales. ${ }^{13}$ En outre, il peut faire partie d'une nouvelle stratégie analgésique permettant la chirurgie ambulatoire de l'épaule (par ex., la réparation de la coiffe des rotateurs) moins laborieuse qu'en milieu hospitalier. Une démarche semblable a été décrite récemment par Rawal dont l'étude préliminaire a démontré que l'administration postopératoire d'anesthésique local contrôlée par le patient à la maison peut être à la fois efficace et sûre dans le cas de nombreuses interventions orthopédiques. ${ }^{14}$ Cependant, avant de souscrire avec enthousiasme à l'utilisation répandue d'anesthésique local à domicile, des données périopératoires qui confirment l'innocuité de la méthode doivent être recueillies par de nouvelles études prospectives à grande échelle.

Généralement acceptée pour les enfants, la réalisation d'un BIS chez des adultes sous anesthésie générale, comme Boezaart la décrit, demeure un sujet de controverse et dépend souvent des critères et des habitudes de pratique de l'institution ou du pays. De récentes données montrent que la paresthésie pendant la mise en place de l'aiguille et la douleur pendant l'injection de l'anesthésique local sont deux éléments-clés étroitement reliés à l'occurrence de lésions neurologiques sérieuses. ${ }^{15}$ Il est donc logique de penser que c'est là un signal d'alarme qui ne sera pas perçu chez un patient inconscient. En outre, des données préliminaires montrent que le blocage nerveux guidé par un neurostimulateur n'est peut-être pas aussi sûr qu'on l'a d'abord cru. Urmey a montré que la paresthésie peut être provoquée sans produire régulièrement des contractions musculaires chez $25 \%$ des patients qui reçoivent une stimulation de $1 \mathrm{~mA}$ pour un BIS, ce qui laisse présager une discordance entre les réponses motrice et sensitive déclenchées par la piqûre de l'aiguille. ${ }^{16}$ Ainsi, une absence de réaction motrice n'écarte pas la présence d'une aiguille près des tissus nerveux. Quelques cas de neuropathie ont été signalés après l'usage de neurostimulateur pour le BIS chez des patients sous anesthésie générale, dont un cas de déficit neurologique permanent. ${ }^{17}$ Selon moi, la décision de procéder à une anesthésie locorégionale chez un patient qui a reçu une forte sédation ou qui est anesthésié ne devrait pas être faite sans distinction. C'est particulièrement vrai pour des interventions comme une arthroplastie complète de l'épaule où une lésion chirurgicale du plexus brachial demeure une complication toujours possible. L'anesthésiologiste n'est alọs plus à l'abri des poursuites.

En résumé, le BIS continu est une technique utile pour fournir une analgésie régionale dans une variété de pathologies de l'épaule. La nouvelle technique décrite par Boezaart améliorera probablement la précision de la mise en place du cathéter du BIS et diminuera le risque de déplacement. Cependant, on ne peut approuver la pratique d'un BIS de routine chez des patients sous anesthésie générale.

\section{References}

1 Tuominen $M$, Haasio J, Hekali $R$, Rosenberg $P H$. Continuous interscalene brachial plexus block: clinical efficacy, technical problems and bupivacaine plasma concentrations. Acta Anaesthesiol Scand 1989; 33: 84-8.

2 Boezaart AP, de Beer JF, du Toit C, van Rooyen K. A new technique of continuous interscalene nerve block. Can J Anesth 1999; 46: 275-81.

3 Tuominen $M K$, Pere $P$, Rosenberg $P H$. Unintentional arterial catheterization and bupivacaine toxity associated with continuous interscalene brachial plexus block. Anesthesiology 1991; 75: 356-8.

4 Cook $L B$. Unsuspected extradural catheterization in an interscalene block. Br J Anaesth 1991; 67: 473-5.

5 Smith MP, Tetzlaff JE, Brems JJ. Asymptomatic profound oxyhemoglobin desaturation following interscalene block in a geriatric patient. Regional Anesthesia and Pain Medicine 1998; 23: 210-3.

6 Urmey WF, Talts KH, Sharrock NE. One hundred percent incidence of hemidiaphragmatic paresis associated with interscalene brachial plexus anesthesia as diagnosed by ultrasonography. Anesth Analg 1991; 72: 498-503.

7 Pere $P$, Pitkanen $M$, Rosenberg $P H$, et al. Effect of continuous interscalene brachial plexus block on diaphragmatic motion and on ventilatory function. Acta Anaesthesiol Scand 1992; 36: 53-7.

8 Al-Kaisy AA, Cban VWS, Perlas A. Respiratory effects of low dose bupivacaine interscalene block. International Monitor on Regional Anaesthesia 1997; 5: 9-3.

9 Al-Kaisy AA, McGuire G, Chan VWS, at al. Analgesic effect of interscalene block using low-dose bupivacaine for outpatient arthroscopic shoulder surgery. Regional Anesthesia and Pain Medicine 1998; 23: 469-73.

10 Al-Kaisy AA, Chan V, Peng P, Perlas A, Miniaci $A$. Postoperative analgesic effect of low dose bupivacaine for interscalene brachial plexus block for shoulder surgery, a dose finding study. Can J Anaesth 1997; 44: A60-A.

11 Borgeat A, Schappi B, Biasca N, Gerber C. Patient-controlled analgesia after major shoulder surgery: patientcontrolled interscalene analgesia vs patient-controlled analgesia. Anesthesiology 1997; 87: 1343-7.

12 Borgeat A, Tewes E, Biasca N, Gerber C. Patient-controlled interscalene analgesia with ropivacaine after major shoulder surgery: PCIA vs PCA. Br J Anaesth 1998; 81: 603-5. 
13 Sato $S$, Yamashita S, Iwai M, Mizuyama $K$, Satsuame $T$. Continuous interscalene block for cancer pain (Letter). Reg Anesth 1994; 19: 73-5.

14 Rawal N, Axelsson K, Hylander J, et al. Postoperative patient-controlled local anesthetic administration at home. Anesth Analg 1998; 86: 86-9.

15 Auroy $\Upsilon$, Narchi P, Messiah A, Litt L, Rouvier B, Sarnii $K$. Serious complications related to regional anesthesia: results of a prospective survey in France Anesthesiology 1997; 87: 479-86.

16 Urmey WF, Stanton J, O=Brien S, Tagariello $V$,

Wickiewicz TL. Inability to consistently elicit a motor response following sensory paresthesia during interscalene block administration. Reg Anesth and Pain Med 1998; 23: S7.

17 Passannante $A$. Spinal anesthesia and permanent neurologic deficit after interscalene block. Anesth Analg 1996; 82: 873-4. 